\title{
Perspective sur les ruptures d'approvisionnement de médicaments en établissement de santé de 2006 à 2010
}

\author{
Jean-François Bussières, Andrei Chiveri et Denis Lebel
}

\section{RÉSUMÉ}

Contexte : Si les ruptures d'approvisionnement de médicaments font partie de la réalité de la pratique pharmaceutique depuis plusieurs décennies, elles deviennent une préoccupation quotidienne pour les pharmaciens dans les années 2000 et dépassent les frontières de la littérature pharmaceutique.

Objectif : Lobjectif principal de cette étude était de quantifier le nombre de médicaments en rupture de stock par année et la durée de ces interruptions. Lobjectif secondaire visait à décrire le nombre de médicaments en rupture de stock par fabricant et par classe thérapeutique.

Méthode : Cette étude descriptive et rétrospective des ruptures d'approvisionnement en médicaments a porté sur l'ensemble des médicaments à contrat pour les hôpitaux des régions administratives de Montréal, de Laval et de l'Estrie, au Québec. Le nombre de ruptures de stocks, le nombre de jours de rupture de stock et leur durée moyenne par année ont été calculés par fabricant et par classe thérapeutique. De plus, la proportion de produits en rupture de stock et la proportion de jours de rupture de stocks ont été déterminées par classe thérapeutique. Les données ont été analysées à l'aide de statistiques descriptives (c.-à-d. somme, moyenne, écart-type, médiane, intervalle).

Résultats : Entre le 1 1er janvier 2006 et le 31 août 2010 (une période de 56 mois), 2400 ruptures de stocks ont été dénombrées pour un total de 258 105 jours de rupture de stocks (durée moyenne de 108 jours, écart-type de 130 jours et intervalle de 5 à 1623 jours). Un total de 70 fabricants étaient impliqués dans la survenue de toutes les ruptures d'approvisionnement de médicaments relevées durant cette période. Cinquante pour cent (50\%) des ruptures de stocks et des jours de rupture de stocks provenaient de quatre fabricants. Les interruptions étudiées touchaient la majorité des classes thérapeutiques de médicaments disponibles sur le marché. Toutefois, $50 \%$ des ruptures de stocks provenaient de trois classes thérapeutiques (médicaments du système nerveux central, agents anti-infectieux et médicaments cardiovasculaires).

Conclusion : Il s'agit des premières données canadiennes publiées sur l'étendue des ruptures d'approvisionnement sur le marché hospitalier. Létude a démontré que les ruptures de stocks touchent la plupart des fabricants et la majorité des classes thérapeutiques. D'autres études sont nécessaires afin d'explorer les causes et les conséquences des ruptures d'approvisionnement en établissements de santé.

Mots clés : ruptures d'approvisionnement en médicaments, rupture de stock, fabricant, établissement de santé

\begin{abstract}
Background: Drug shortages have been a reality of pharmacy practice for decades. However, this problem has become a daily concern for pharmacists in the 21st century and extends beyond the pharmaceutical literature.
\end{abstract}

Objective: The primary objective of this study was to quantify the annual number of drugs in short supply and the duration of these shortages. A secondary objective was to describe the number of drugs in short supply by manufacturer and therapeutic class.

Method: This descriptive retrospective study examined drug shortages in relation to all hospital medication supply contracts in the administrative regions of Montréal, Laval, and the Eastern Townships in the province of Quebec. The number of inventory shortages, the number of out-of-stock days, and the mean annual duration of the shortages were calculated by manufacturer and by therapeutic class. The proportions of out-of-stock products and out-of-stock days were also calculated by therapeutic class. Descriptive statistical analyses were performed (i.e., totals, means, standard deviations, medians, and ranges).

Results: There were 2400 inventory shortages between January 1, 2006, and August 31, 2010 (56-month period) for a total of 258105 out-ofstock days (mean duration \pm standard deviation $108 \pm 130$ days, range 5 to 1623 days). A total of 70 manufacturers were implicated in drug shortages over the study period, but 4 manufacturers were responsible for half $(50 \%)$ of the shortages and out-of-stock days. The shortages affected the majority of therapeutic drug classes on the market. However, 3 therapeutic classes (central nervous system drugs, anti-infective agents, and cardiovascular drugs) accounted for $50 \%$ of the shortages.

Conclusion: These are the first Canadian data published on the scope of drug shortages in the hospital market. This study has demonstrated that drug shortages affect the majority of manufacturers and most therapeutic classes. Further studies are required to explore the causes and effects of drug shortages in the hospital setting.

Key words: drug shortages, inventory shortage, manufacturer, hospital

[Publisher's translation] 


\section{INTRODUCTION}

$\mathrm{O}$ recense les premiers écrits sur les ruptures d'approvisionnement de médicaments dans les années soixante$\mathrm{dix}^{1,2}$. Si les ruptures d'approvisionnement de médicaments font partie de la réalité de la pratique pharmaceutique depuis plusieurs décennies ${ }^{3,4}$ elles deviennent une préoccupation quotidienne des pharmaciens dans les années deux mille et dépassent les frontières de la littérature pharmaceutique ${ }^{5-9}$. Bien que le problème ait été davantage décrit en Amérique du Nord, il touche aussi d'autres pays européens ${ }^{10}$. La Food and Drug Administration (FDA) publie un portail sur le sujet ${ }^{11}$. L'American Society of Health-System Pharmacists (ASHP) a publié en 2001 et en 2009 des lignes directrices sur le sujet ${ }^{12,13}$ et a mis en place un portail comportant un formulaire de déclaration en ligne des ruptures d'approvisionnement ${ }^{14}$. Au Canada, l'Association des pharmaciens du Canada a publié en septembre 2010 un guide pour l'évaluation et la gestion des patients en présence de ruptures d'approvisionnement ${ }^{15}$. De plus, un site web informatif de l'Unité de recherche en pratique pharmaceutique du CHU Sainte-Justine a été mis en ligne en février $2011^{16}$. Au Québec, en vertu de la Loi sur les contrats des organismes publics ${ }^{17}$, Sigma Santé représente les établissements de santé aux fins de négocier une entente sur les médicaments. En 2009-2010, la valeur annuelle des achats de médicaments issus de l'entente de groupe était d'environ 240 millions de dollars. Les chefs de départements de pharmacie sont tenus de mandater les quantités annuelles requises de chaque médicament inscrit à leur liste locale. De plus, Sigma Santé négocie une entente de distribution avec un grossiste afin de faciliter la chaîne logistique entre les fabricants et les hôpitaux. Durant la période étudiée, l'attributaire de l'entente de distribution est Mckesson Canada. L'entente de distribution prévoit la publication périodique, tous les quinze jours, d'une liste des produits en rupture d'approvisionnement. Cette liste est diffusée par courriel à tous les chefs de départements de pharmacie. Le personnel de Sigma Santé procède à une consolidation périodique de ces données de rupture de stocks afin de produire un tableau synthèse au comité des usagers piloté par un groupe de pharmaciens élus par la Table des chefs de départements des trois régions administratives concernées. Les données portant sur les ruptures d'approvisionnement de médicaments sont recueillies par Sigma Santé depuis 2003; toutefois, des données sont recueillies de façon plus systématique depuis 2006. Lobjectif de cet article est de déterminer l'envergure des ruptures d'approvisionnement en établissement de santé au Québec.

\section{MÉTHODE}

Il s'agit d'une étude descriptive et rétrospective des ruptures d'approvisionnement en médicaments. L'objectif principal vise à quantifier le nombre de médicaments en rupture de stocks par année et la durée de ces interruptions. Lobjectif secondaire consiste à décrire le nombre de médicaments en rupture de stocks par fabricant et par classe thérapeutique.

Létude porte sur l'ensemble des médicaments à contrat avec Sigma Santé (auparavant Approvisionnements Montréal) pour les hôpitaux des régions administratives de Montréal, de Laval et de l'Estrie. L'étude porte sur l'ensemble des ruptures de

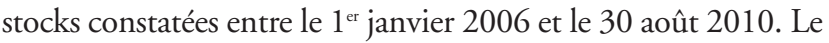
fichier de données fourni par Sigma Santé comporte les variables suivantes : nom du fabricant, numéro de catalogue Sigma Santé, numéro de produit Mckesson, classe thérapeutique, nom générique, nom commercial, y compris la teneur et la forme, la date de début et de fin, la rupture de stock et le retour en stock ou le retrait de marché.

Nous avons consolidé les données recueillies dans une feuille de travail d'un chiffrier électronique (Microsoft Excel, Suite Office 2007, Seattle [Washington]) de sorte que chaque épisode de rupture d'approvisionnement ne comporte qu'une ligne de données et une seule date de début et de fin. Les données ont été validées et croisées avec celles du catalogue de produits de McKesson, des ententes d'achats groupés et de la banque de données des produits pharmaceutiques (BDPP) de Santé Canada pour la conformité des descriptifs de nom générique, des classes thérapeutiques, des numéros d'identification de médicament (DIN) et des numéros d'ingrédients actifs. Le numéro d'ingrédient actif permet d'apparier chaque produit en rupture de stock à toutes les solutions de remplacement de même teneur disponibles dans la BDPP.

Nous avons calculé le nombre de ruptures de stocks, le nombre de jours de ruptures de stocks (p. ex. pour un produit donné, 10 jours-rupture correspondent à une rupture de stocks de 10 jours consécutifs), la durée moyenne des ruptures d'approvisionnement de médicaments par année, par fabricant et par classe thérapeutique, selon la classification de l'American Hospital Formulary Service. Dans le cas des ruptures d'approvisionnement dont la fin n'avait pas été confirmée au 31 août 2010, nous avons utilisé cette date pour le calcul de la durée. Dans le cas des ruptures d'approvisionnement échelonnées sur plus d'une année civile, nous avons tenu compte de la durée totale de la rupture en nombre de jours. De plus, nous avons calculé la proportion de produits en rupture de stock (c.-à-d. le nombre de produits en rupture de stocks par classe thérapeutique par rapport au nombre de produits disponibles de la classe dans la BDPP au 31 août 2010) et la proportion de jours-rupture par classe thérapeutique (c.-̀̀-d. le nombre de jours-rupture par classe thérapeutique par rapport au nombre total de produits de la classe disponibles dans la BDPP au 31 août 2010 multiplié par 365 jours). 
Nous n’avons effectué que des analyses statistiques descriptives (c.-à-d. somme, moyenne, écart-type, médiane, intervalle).

\section{RÉSULTATS}

Notre étude a permis d'enregistrer un total de 2400 ruptures de stocks du $1^{\text {er }}$ janvier 2006 au 31 août 2010 pour un total de 258105 jours-rupture (durée moyenne \pm écart-type de $108 \pm 130$ jours; intervalle de 5 à 1623) répartis comme suit : 493 ruptures de stocks et 32693 jours-rupture en 2006, 400 ruptures de stocks et 38137 jours-rupture en 2007, 442 ruptures de stocks et 40162 jours-rupture en 2008, 680 ruptures de stocks et 68512 jours-rupture en 2009, 385 ruptures de stocks et 78601 jours-rupture de janvier à août 2010 .

Un total de 70 fabricants sont impliqués dans la survenue de toutes les ruptures d'approvisionnement de médicaments recensées durant la période étudiée. Cinquante pour cent (50\%) des ruptures de stocks et des jours-rupture proviennent de quatre fabricants. Vingt-six (26) des 70 fabricants sont responsables d'au moins 16 ruptures d'approvisionnement durant la période étudiée. Le tableau 1 présente le profil des ruptures d'approvisionnement de médicaments par fabricant du $1^{\text {er }}$ janvier 2006 au 31 août 2010.

L'ensemble des ruptures de stocks étudiées touche la majorité des classes thérapeutiques disponibles sur le marché. Toutefois, $50 \%$ des ruptures de stocks proviennent de trois classes thérapeutiques soit 28:00 - médicaments du système nerveux central (588 ruptures, 58572 jours-rupture), 08:00 anti-infectieux (322 ruptures, 37408 jours-rupture) et 24:00 médicaments cardiovasculaires (285 ruptures, 35538 joursrupture). Le tableau 2 présente le profil des ruptures d'approvisionnement de médicaments par classe thérapeutique pendant la durée de l'étude. Le profil précise la proportion de produits en rupture de stocks de chaque classe thérapeutique et la proportion de jours-rupture. Treize (13) classes thérapeutiques comportent plus de $10 \%$ des produits en rupture de stocks et plus de $30 \%$ de produits en rupture de stocks sont notés dans les classes « antidotes des métaux lourds " (64:00) et « vaccins» $(80: 00)$.

Tableau 1. Profil des ruptures d'approvisionnement de médicaments par fabricant du

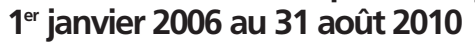

\begin{tabular}{|c|c|c|c|}
\hline Fabricant & $\begin{array}{l}\text { Nombre de ruptures de } \\
2006 \text { à } 2010\end{array}$ & $\begin{array}{c}\text { Nombre de } \\
\text { jours-rupture }\end{array}$ & $\begin{array}{l}\text { Durée moyenne } \pm \\
\text { écart-type (jours) }\end{array}$ \\
\hline Apotex Inc & 453 & 58707 & $130 \pm 135$ \\
\hline Pharmascience & 330 & 28551 & $87 \pm 77$ \\
\hline Novopharm & 292 & 33493 & $115 \pm 142$ \\
\hline Sandoz & 238 & 21445 & $90 \pm 129$ \\
\hline Hospira & 137 & 13491 & $98 \pm 91$ \\
\hline Teva & 97 & 12905 & $133 \pm 124$ \\
\hline Baxter & 77 & 7618 & $99 \pm 123$ \\
\hline Ratiopharm & 76 & 6250 & $82 \pm 60$ \\
\hline Omega & 56 & 4820 & $86 \pm 66$ \\
\hline Taro & 47 & 6914 & $147 \pm 185$ \\
\hline GlaxoSmithKline & 42 & 2851 & $68 \pm 55$ \\
\hline Abbott Laboratoires & 41 & 2247 & $55 \pm 37$ \\
\hline Genpharm & 40 & 4234 & $106 \pm 107$ \\
\hline Valeant & 35 & 4093 & $117 \pm 104$ \\
\hline Sanofi-Aventis Pharma & 32 & 3702 & $116 \pm 143$ \\
\hline Pfizer & 28 & 2548 & $91 \pm 81$ \\
\hline Erfa & 27 & 7588 & $281 \pm 326$ \\
\hline Janssen & 25 & 1703 & $68 \pm 58$ \\
\hline Partenaires Pharma & 22 & 1546 & $70 \pm 76$ \\
\hline Bristol-Myers Squibb & 21 & 1353 & $64 \pm 33$ \\
\hline AA Pharma & 20 & 3896 & $195 \pm 123$ \\
\hline Schering & 20 & 2366 & $118 \pm 157$ \\
\hline Euro-Pharm & 19 & 3654 & $192 \pm 360$ \\
\hline Squire & 18 & 3420 & $190 \pm 292$ \\
\hline Eli Lilly & 16 & 626 & $39 \pm 20$ \\
\hline Wyeth Pharma & 16 & 1170 & $73 \pm 62$ \\
\hline $\begin{array}{l}44 \text { fabricants annonçant } 15 \text { ruptures de stocks } \\
\text { ou moins pendant la période de l'étude }\end{array}$ & 175 & 16914 & $86 \pm 76$ \\
\hline Total (70 fabricants) & 2400 & 258105 & $\begin{array}{l}108 \pm 130 * \\
(5-1623)\end{array}$ \\
\hline
\end{tabular}

*Pour tous les produits, y compris ceux encore en rupture de stock le 30 août 2010. 
Tableau 2. Profil des ruptures d'approvisionnement de médicaments par classe thérapeutique du 1er janvier 2006 au 31 août 2010

\begin{tabular}{|c|c|c|c|c|}
\hline Classe thérapeutique & Nombre de ruptures & Nombre de jours-rupture & $\%$ de produits en rupture* & $\%$ jours-rupturet \\
\hline \multicolumn{5}{|l|}{ Médicaments S.N.C. } \\
\hline 2006 & 124 & 10701 & 14 & 3 \\
\hline 2007 & 86 & 7680 & 8 & 2 \\
\hline 2008 & 99 & 10230 & 9 & 3 \\
\hline 2009 & 169 & 19162 & 19 & 6 \\
\hline 2010キ & 110 & 10799 & 13 & 3 \\
\hline Somme ou moyenne§ & 588 & 58572 & 12,6 & 3,4 \\
\hline \multicolumn{5}{|c|}{ Médicaments cardiovasculaires } \\
\hline 2006 & 44 & 6178 & 9 & 4 \\
\hline 2007 & 44 & 3740 & 8 & 2 \\
\hline 2008 & 48 & 6125 & 9 & 3 \\
\hline 2009 & 95 & 14191 & 20 & 8 \\
\hline 2010キ & 54 & 5304 & 12 & 3 \\
\hline Somme ou moyenne§ & 285 & 35538 & 11,6 & 4,0 \\
\hline \multicolumn{5}{|l|}{ Anti-infectieux } \\
\hline 2006 & 56 & 5370 & 13 & 3 \\
\hline 2007 & 46 & 4415 & 9 & 2 \\
\hline 2008 & 53 & 6883 & 10 & 4 \\
\hline 2009 & 118 & 15593 & 28 & 10 \\
\hline $2010 \neq$ & 49 & 5147 & 13 & 4 \\
\hline Somme ou moyenne§ & 322 & 37408 & 14,6 & 4,6 \\
\hline \multicolumn{5}{|c|}{ Médicaments gastrointestinaux } \\
\hline 2006 & 34 & 2625 & 13 & 3 \\
\hline 2007 & 20 & 1501 & 6 & 1 \\
\hline 2008 & 26 & 2587 & 8 & 2 \\
\hline 2009 & 47 & 4111 & 19 & 4 \\
\hline 2010キ & 25 & 2360 & 11 & 3 \\
\hline Somme ou moyenne§ & 152 & 13184 & 11,4 & 2,6 \\
\hline \multicolumn{5}{|c|}{ Électrolytes-diurétiques } \\
\hline 2006 & 38 & 4813 & 17 & 6 \\
\hline 2007 & 28 & 1751 & 11 & 2 \\
\hline 2008 & 30 & 2220 & 12 & 2 \\
\hline 2009 & 36 & 4290 & 18 & 6 \\
\hline $2010 \neq$ & 23 & 2159 & 13 & 3 \\
\hline Somme ou moyenne§ & 155 & 15233 & 14,2 & 3,8 \\
\hline \multicolumn{5}{|l|}{ Peau et muqueuse } \\
\hline 2006 & 35 & 4438 & 10 & 3 \\
\hline 2007 & 36 & 2885 & 8 & 2 \\
\hline 2008 & 20 & 4759 & 4 & 3 \\
\hline 2009 & 27 & 4462 & 8 & 4 \\
\hline 2010キ & 22 & 2111 & 7 & 2 \\
\hline Somme ou moyenne§ & 140 & 18655 & 7,4 & 2,8 \\
\hline \multicolumn{5}{|l|}{ Non-classés } \\
\hline 2006 & 34 & 4994 & 6 & 2 \\
\hline 2007 & 31 & 2492 & 5 & 1 \\
\hline 2008 & 27 & 3157 & 4 & 1 \\
\hline 2009 & 41 & 4482 & 8 & 2 \\
\hline $2010 \neq$ & 20 & 2219 & 4 & 1 \\
\hline Somme ou moyenne§ & 153 & 17344 & 5,4 & 1,4 \\
\hline
\end{tabular}


Tableau 2. Profil des ruptures d'approvisionnement de médicaments par classe thérapeutique du 1ªr janvier 2006 au 31 août 2010 (suite)

\begin{tabular}{|c|c|c|c|c|}
\hline Classe thérapeutique & Nombre de ruptures & Nombre de jours-rupture & $\%$ de produits en rupture* & $\%$ jours-rupturet \\
\hline \multicolumn{5}{|l|}{ Médicaments S.N.A. } \\
\hline 2006 & 27 & 2084 & 14 & 3 \\
\hline 2007 & 23 & 2188 & 10 & 3 \\
\hline 2008 & 35 & 2918 & 16 & 4 \\
\hline 2009 & 32 & 3649 & 18 & 6 \\
\hline $2010 \neq$ & 18 & 1851 & 11 & 3 \\
\hline Somme ou moyenne§ & 135 & 12690 & 13,8 & 3,8 \\
\hline \multicolumn{5}{|l|}{ Médicaments O.R.L.O. } \\
\hline 2006 & 22 & 1547 & 12 & 2 \\
\hline 2007 & 12 & 677 & 6 & 1 \\
\hline 2008 & 23 & 6619 & 11 & 9 \\
\hline 2009 & 16 & 1851 & 9 & 3 \\
\hline $2010 \neq$ & 17 & 1248 & 10 & 2 \\
\hline Somme ou moyenne§ & 90 & 11942 & 9,6 & 3,4 \\
\hline \multicolumn{5}{|l|}{ Antinéoplasiques } \\
\hline 2006 & 13 & 741 & 9 & 1 \\
\hline 2007 & 14 & 1966 & 7 & 3 \\
\hline 2008 & 19 & 2771 & 10 & 4 \\
\hline 2009 & 21 & 2775 & 13 & 5 \\
\hline 2010キ & 15 & 1073 & 10 & 2 \\
\hline Somme ou moyenne§ & 82 & 9326 & 9,8 & 3,0 \\
\hline \multicolumn{5}{|l|}{ Médicaments du sang } \\
\hline 2006 & 18 & 1176 & 12 & 2 \\
\hline 2007 & 11 & 713 & 6 & 1 \\
\hline 2008 & 21 & 2428 & 12 & 4 \\
\hline 2009 & 18 & 1596 & 11 & 3 \\
\hline 2010キ & 12 & 712 & 8 & 1 \\
\hline Somme ou moyenne§ & 80 & 6625 & 9,8 & 2,2 \\
\hline \multicolumn{5}{|c|}{ Hormones et substituts } \\
\hline 2006 & 20 & 1872 & 8 & 2 \\
\hline 2007 & 21 & 2497 & 7 & 2 \\
\hline 2008 & 21 & 2262 & 7 & 2 \\
\hline 2009 & 32 & 3414 & 13 & 4 \\
\hline 2010キ & 10 & 763 & 4 & 1 \\
\hline Somme ou moyenne§ & 104 & 10808 & 7,8 & 2,2 \\
\hline \multicolumn{5}{|l|}{ Antihistaminiques } \\
\hline 2006 & 1 & 158 & 3 & 1 \\
\hline 2007 & 3 & 180 & 7 & 1 \\
\hline 2008 & 6 & 851 & 14 & 5 \\
\hline 2009 & 5 & 617 & 14 & 5 \\
\hline 2010キ & 5 & 536 & 16 & 5 \\
\hline Somme ou moyenne§ & 20 & 2342 & 10,8 & 3,4 \\
\hline \multicolumn{5}{|l|}{ Autres } \\
\hline 2006 & 13 & 770 & 17 & 3 \\
\hline 2007 & 13 & 949 & 15 & 3 \\
\hline 2008 & 8 & 550 & 9 & 2 \\
\hline 2009 & 7 & 602 & 11 & 3 \\
\hline $2010 \neq$ & 2 & 67 & 3 & 0 \\
\hline Somme ou moyenne§ & 43 & 2938 & 11,0 & 2,2 \\
\hline
\end{tabular}


Tableau 2. Profil des ruptures d'approvisionnement de médicaments par classe thérapeutique du 1er janvier 2006 au 31 août 2010 (suite)

\begin{tabular}{|c|c|c|c|c|}
\hline Classe thérapeutique & Nombre de ruptures & Nombre de jours-rupture & $\%$ de produits en rupture* & $\%$ jours-rupturet \\
\hline \multicolumn{5}{|l|}{ Ocytociques } \\
\hline 2006 & 0 & 0 & 0 & 0 \\
\hline 2007 & 0 & 0 & 0 & 0 \\
\hline 2008 & 0 & 0 & 0 & 0 \\
\hline 2009 & 0 & 0 & 0 & 0 \\
\hline 2010キ & 1 & 113 & 8 & 2 \\
\hline Somme ou moyenne§ & 1 & 113 & 1,6 & 0,4 \\
\hline \multicolumn{5}{|l|}{ Anésthétiques locaux } \\
\hline 2006 & 4 & 119 & 8 & 1 \\
\hline 2007 & 6 & 274 & 10 & 1 \\
\hline 2008 & 1 & 154 & 2 & 1 \\
\hline 2009 & 7 & 1017 & 14 & 6 \\
\hline 2010キ & 1 & 104 & 2 & 1 \\
\hline Somme ou moyenne§ & 19 & 1668 & 7,2 & 2,0 \\
\hline \multicolumn{5}{|l|}{ Agents diagnostiques } \\
\hline 2006 & 4 & 387 & 21 & 6 \\
\hline 2007 & 5 & 353 & 21 & 4 \\
\hline 2008 & 0 & 0 & 0 & 0 \\
\hline 2009 & 1 & 134 & 6 & 2 \\
\hline $2010 \neq$ & 1 & 40 & 6 & 1 \\
\hline Somme ou moyenne§ & 11 & 914 & 10,8 & 2,6 \\
\hline \multicolumn{5}{|l|}{ Matériels } \\
\hline 2006 & 4 & 629 & 10 & 4 \\
\hline 2007 & 1 & 100 & 3 & 1 \\
\hline 2008 & 2 & 52 & 5 & 0 \\
\hline 2009 & 4 & 723 & 12 & 6 \\
\hline 2010キ & 0 & 0 & 0 & 0 \\
\hline Somme ou moyenne§ & 11 & 1504 & 6,0 & 2,2 \\
\hline \multicolumn{5}{|c|}{ Antidotes des métaux lourds } \\
\hline 2006 & 0 & 0 & 0 & 0 \\
\hline 2007 & 0 & 0 & 0 & 0 \\
\hline 2008 & 1 & 144 & 17 & 7 \\
\hline 2009 & 2 & 192 & 44 & 12 \\
\hline $2010 \neq$ & 0 & 0 & 0 & 0 \\
\hline Somme ou moyenne§ & 3 & 336 & 12,2 & 3,8 \\
\hline \multicolumn{5}{|l|}{ Vaccins } \\
\hline 2006 & 1 & 83 & 33 & 8 \\
\hline 2007 & 0 & 0 & 0 & 0 \\
\hline 2008 & 1 & 184 & 33 & 17 \\
\hline 2009 & 0 & 0 & 0 & 0 \\
\hline 2010キ & 0 & 0 & 0 & 0 \\
\hline Somme ou moyenne§ & 2 & 267 & 13,2 & 5,0 \\
\hline \multicolumn{5}{|c|}{$\begin{array}{l}\text { Antitussifs, expectorants et } \\
\text { agents mucolytiques }\end{array}$} \\
\hline 2006 & 1 & 100 & 6 & 2 \\
\hline 2007 & 0 & 0 & 0 & 0 \\
\hline 2008 & 0 & 0 & 0 & 0 \\
\hline 2009 & 1 & 148 & 6 & 3 \\
\hline 2010キ & 0 & 0 & 0 & 0 \\
\hline Somme ou moyenne§ & 2 & 248 & 2,4 & 1,0 \\
\hline
\end{tabular}


This single copy is for your personal, non-commercial use only.

For permission to reprint multiple copies or to order presentation-ready copies for distribution, contact CJHP at cjhpedit@cshp.ca

\section{Tableau 2. Profil des ruptures d'approvisionnement de médicaments par classe thérapeutique du $1^{\text {er janvier } 2006}$ au 31 août 2010 (suite et fin)}

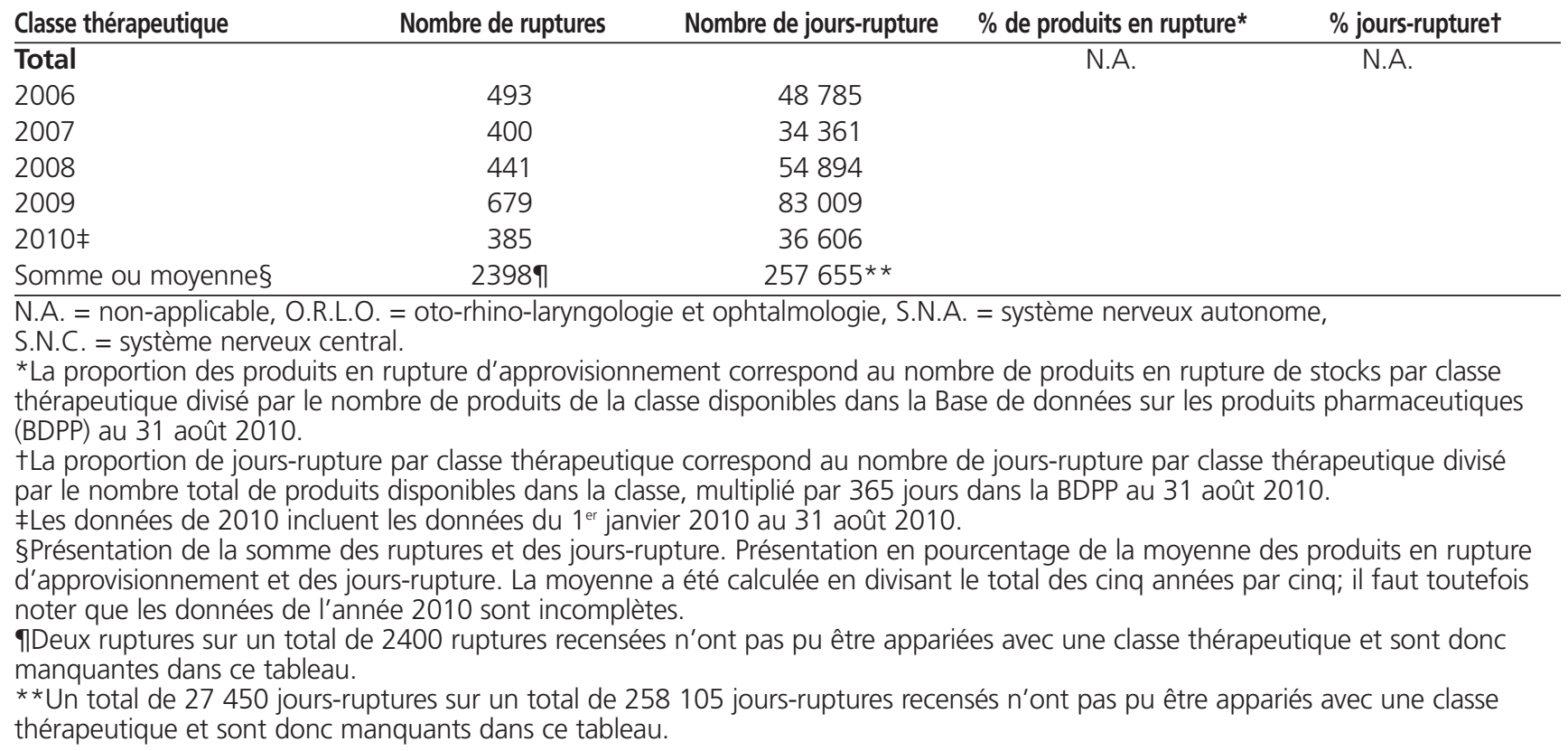

\section{DISCUSSION}

La problématique des ruptures d'approvisionnement fait l'objet d'une couverture médiatique sans précédent au cours des derniers mois, tant aux États-Unis qu'au Canada ${ }^{18-23}$. Ces reportages font ressortir une augmentation du nombre et du caractère préoccupant des ruptures de stocks, tant pour les patients que pour les professionnels de la santé concernés. Le plus souvent, une rupture d'approvisionnement de médicaments ne constitue pas une menace pour la santé des patients, parce qu'il existe une solution de remplacement chez un autre fabricant ou une autre teneur de la même entité générique chez le fabricant à l'origine de la rupture de stock. Toutefois, plusieurs ruptures d'approvisionnement récentes ne comportent aucune solution de substitution (p. ex. hydralazine, cytarabine, thiopental, dacarbazine, succinylcholine, etc.). En outre, on reconnaît que la problématique des ruptures d'approvisionnement de médicaments est multifactorielle et qu'elle survient à toutes les étapes du circuit du médicament, de la production de la matière première, en passant par la fabrication du produit commercialisé par le manufacturier, la distribution du produit commercialisé par les grossistes, la négociation des produits commercialisés par les regroupements d'achats et les achats de ces produits par les établissements de santé et les pharmacies d'officine ${ }^{24}$.

Notre étude révèle un nombre annuel élevé et soutenu de ruptures d'approvisionnement de médicaments depuis 2006, soit 493 en 2006, 400 en 2007, 442 en 2008, 680 en 2009 et 385 en 2010. En extrapolant les données de 2010 pour une durée de 12 mois, on peut estimer un total de plus de 550 ruptures de stocks. Il existe peu de données sur le nombre de produits en rupture d'approvisionnement. Fox et Tyler ont recensé 224 ruptures d'approvisionnement au sein des University of Utah Hospitals and Clinics de janvier 1996 à juin $2002^{25}$. De ces 224 ruptures de stocks, 119 sont survenues en 2001 et 70 de ces 119 étaient encore non résolues au terme de l'étude. Dans un éditorial, Fox et Tyler ont souligné la présence de 66 ruptures d'approvisionnement actives sur le marché américain au $1^{\text {er }}$ juin 2004, dont 55 qui duraient depuis plus d'un $\mathrm{an}^{26}$. Fox et Tyler ont monitoré 150 ruptures d'approvisionnement en 2008 à partir de leur centre d'information consacré, notamment, au suivi des ruptures de stocks pour le portail de l'ASHP27. Les données issues du programme de monitorage des ruptures d'approvisionement en médicaments de la FDA mentionnent un total de 157 ruptures en 2009 , dont $46 \%$ sont des produits stériles ${ }^{28}$. Au Québec, le Conseil du médicament mentionne dans son rapport de gestion de 2009-2010, 61 avis de rupture d'approvisionnement touchant 72 médicaments envoyés à la Régie de l'assurance maladie du Québec (RAMQ) ${ }^{29}$. Sur le site “Vendredi PM » on recense en date du 23 août 2011, 374 produits en rupture active de stocks pour l'année 2010-2011 ${ }^{16}$. Il est difficile de comparer nos données à celles publiées, compte tenu des motifs qui suivent. Certains auteurs dénombrent les ruptures de stocks par nom de commerce, par DIN (au Canada, un DIN correspond à un ou à plusieurs formats d'une même teneur d'une même forme - p. ex. le DIN 0065759 représente tous les formats d'Apo-lorazépam en comprimés de $1 \mathrm{mg}$, soit les 
formats de 30,100, 500 et 1000 comprimés) ou par numéros de produits attribués par le fabricant et le grossiste. Nos données dénombrent les ruptures de stocks par numéros de produits, ce qui reflète davantage l'étendue des produits non disponibles. Au $1^{\text {er }}$ avril 2010, Santé Canada indique que la BDPP contient plus de 18000 produits commercialisés. De ce marché, on peut dire que le nombre de ruptures de stocks enregistrées dans notre étude représente environ $3 \%$ des produits commercialisés. Treize (13) classes thérapeutiques comportent plus de $10 \%$ des produits en rupture de stocks et plus de $30 \%$ de produits en rupture de stocks sont notés dans les classes « antidotes des métaux lourds » et « vaccins ». À notre connaissance, les données publiées sur les ruptures de stocks ne font généralement pas référence à la proportion des produits en rupture d'approvisionnement. Nous pensons que cette dimension est importante et que les données absolues (c.-̀̀-d. nombre de produits en rupture de stock) ne suffisent pas à évaluer l'importance de la problématique.

Fait à noter, les logiciels de gestion des ressources matérielles utilisés en établissements de santé ne permettent pas de répertorier facilement les situations de rupture de stocks (c.-à-d. commandes effectuées mais modifiées par l'usager compte tenu des ruptures de stocks).

Notre étude révèle une durée moyenne de 108 jours par rupture d'approvisionnement, soit une durée variant de 5 à 1623 jours de rupture de stock. Ces données se rapprochent de celles de Fox et coll., qui recensent des durées médianes de 91 à 137 jours de 2003 à $2007^{27}$. Le rapport canadien sur la pharmacie hospitalière de 2009-2010 indique un taux moyen de rotation des stocks de 10,2 en 2009-2010 contre 10,6 en $2007-2008^{30}$. De plus, on note une réduction progressive du taux moyen de rotation en établissement de santé depuis 20052006 (c.-à-d. le taux de rotation était de 10,9) ${ }^{31}$. En vertu de ce taux moyen, les établissements de santé détiennent des stocks pour 36 jours environ de chaque produit, de sorte qu'ils en ressentent les conséquences dans la majorité des cas de ruptures d'approvisionnement, puisque la durée moyenne de ces dernières est plus longue. Les pharmaciens d'établissements sont sans doute tentés de grossir leurs stocks pour limiter les conséquences des pénuries de médicaments.

Notre étude révèle que quatre fabricants se partagent la responsabilité de $50 \%$ des ruptures de stocks. Ceci n'est pas étonnant compte tenu de la gamme étendue de produits génériques commercialisés par Apotex, Pharmascience, Novopharm et Sandoz. La mention distincte de la compagnie Teva s'explique par son acquisition de Novopharm durant la période étudiée. Lévaluation des ruptures de stocks tient compte du nom du fabricant au moment de la survenue de la rupture d'approvisionnement. Outre la gamme élargie de produits provenant de ces quatre fabricants, notons les problèmes de conformité de la société Apotex sur le marché américain ${ }^{32,33}$. Nous comprenons que les correctifs exigés par la FDA ont contribué à la revalidation de procédés et de pratiques et à la survenue de nombreuses ruptures de stocks. Apotex a été réautorisé à vendre des produits sur le marché américain en mai $2011^{34}$.

Notre étude révèle que la majorité des classes thérapeutiques disponibles sur le marché sont visées par des ruptures. Il existe peu de données portant sur la représentation relative des classes thérapeutiques. Les ruptures de stocks sont le plus souvent décrites par dénomination commune de produits plutôt que par classes thérapeutiques ${ }^{27}$. Nos données confirment l'étendue de la problématique des ruptures d'approvisionnement de médicaments et la difficulté pour les professionnels de la santé d'accorder la priorité à certaines de leurs actions afin de prévenir l'épuisement des réserves.

Devant l'ampleur du phénomène, on assiste à une mobilisation sans précédent, tant au niveau politique (le sénat américain propose une législation encadrant les ruptures d'approvisionnement ${ }^{35}$ ) que médical ${ }^{1,36}$ ou pharmaceutique. En outre, plusieurs auteurs s'intéressent à l'évaluation des causes $^{37,38}$ et des impacts des ruptures d'approvisionnement ${ }^{8,33,40}$ ainsi que du rôle accru des autorités réglementaires ${ }^{41,42}$. Bien que notre étude n'ait pas pour objectif de déterminer les causes des ruptures, la collecte et l'analyse de ces données portent à réflexion. Si les causes des ruptures d'approvisionnement sont multifactorielles, les pharmaciens d'établissements doivent donc réfléchir aux actions qu'ils peuvent poser afin de les limiter. Nos données, issues des achats groupés, portent à penser que certaines ruptures de produits peuvent être causées par les retours répétés en appels d'offre. En outre, la consolidation (fusion) de groupes d'achat, limitant le nombre de joueurs sur le marché, et les circonstances contractuelles opportunes peuvent contribuer à limiter le nombre de fabricants et à fragiliser le marché.

Cette étude comporte des limites. Les données recueillies reposent sur un fichier périodique de données transmis par un grossiste, dans le cadre de notre entente de distribution de médicaments signée en collaboration avec le groupe d'achat Sigma Santé. Les données recueillies ne reflètent pas forcément toutes les ruptures du marché, et particulièrement celles spécifiques au milieu communautaire. Toutefois, il faut dire que la majorité des médicaments utilisés en soins ambulatoires le sont en établissement de santé, dans la perspective d'un continuum de soins. De plus, la situation qui prévaut au Québec n'est probablement pas différente de celle à l'échelle du pays, étant donné qu'il s'agit des mêmes fabricants et d'un réseau limité de grossistes. Compte tenu du caractère multifactoriel de certaines ruptures, les données analysées excluent les ruptures de stocks liées à des retards de réapprovisionnement par le grossiste. De même, les données excluent les médicaments retirés du marché. Enfin, notre étude 
ne relève que le nombre et la nature des ruptures d'approvisionnement mais pas leurs conséquences sur les professionnels de la santé et les patients. L'évaluation des effets de ces interruptions sur ces personnes devrait tenir compte de différentes variables, notamment la charge de travail et les coûts pour les professionnels de la santé, et les conséquences pour la santé des patients par la prise en compte des événements indésirables potentiels ou avérés.

\section{CONCLUSION}

Cette étude dénombre 2400 ruptures d'approvisionnement de médicaments du $1^{\text {er }}$ janvier 2006 au 30 août 2010 au sein des établissements de santé de la région de Montréal, de Laval, de l'Estrie, au Québec. Il s'agit des premières données canadiennes publiées sur l'étendue des ruptures d'approvisionnement sur le marché hospitalier. L'étude démontre que les ruptures de stocks touchent la majorité des fabricants et la majorité des classes thérapeutiques. D'autres études sont nécessaires afin d'explorer les causes et les impacts des ruptures d'approvisionnement en établissement de santé.

\section{References}

1. Stolar MH. Drug shortages. Science 1976;192(4235):94.

2. Kubica AJ, Bowman G. A statewide drug shortage reporting system [lettre]. Am J Hosp Pharm 1976;33(7):628-631.

3. Myronuk LD, Meagher JB. Drug patent law reform and shortages. $C M A J$ 1993;149(9):1225.

4. Young D. Drug shortages frustrate health care providers. Am J Health Syst Pharm 2002;59(8):698-699,703.

5. Inglis T. National drug shortages. Am J Nurs 2002;102(7):11.

6. Tyler LS, Fox ER, Caravati EM. The challenge of drug shortages for emergency medicine. Ann Emerg Med 2002;40(6):598-602.

7. Eggertson L. Continuing drug shortages affect North American patients. CMAJ 2010;182(18):E811-E812.

8. Steinbrook R. Drug shortages and public health. $N$ Engl J Med 2009;361(16):1525-1527.

9. Traynor K. Drug shortages mount in 2010. Am J Health Syst Pharm 2010;67(18):1492,1494.

10. Ovretveit J, Sachs MA. [Quality and shortages of patient safety—how big is the problem?]. Lakartidningen 2005;102(1-2):45-48. Swedish.

11. Drug shortages. Silverspring (MD) : Food and Drug Administration. Publié au www.fda.gov/drugs/drugsafety/drugshortages/default.htm. Consulté le 25 mai 2011.

12. American Society of Health-System Pharmacists, Council on Administrative Affairs. ASHP guidelines on managing drug product shortages. Am J Health Syst Pharm 2001;58(15):1445-1450.

13. ASHP Expert Panel on Drug Product Shortages; Fox ER, Birt A, James $\mathrm{KB}$, Kokko H, Salverson S, Soflin DL, et al. ASHP guidelines on managing drug product shortages in hospitals and health systems. Am J Health Syst Pharm 2009;66(15):1399-1406.

14. Drug shortage resources center. Bethesda (MD): American Society of Health-System Pharmacists. Publié au www.ashp.org/shortages. Consulté le 6 mai 2011.

15. Drug shortages. A guide for assessment and and patient management. Ottawa $(\mathrm{ON})$ : Association des pharmaciens du Canada; 2010. Publié au www.pharmacists.ca/content/hcp/resource_centre/practice_resources/pdf /Drug\%20Shortages_Sept2010.pdf. Consulté le 11 mai 2011.

16. Ruptures d'approvisionnement en médicaments au Canada. Montréal (QC) : CHU Sainte-Justine, Unité de recherche en pratique pharmaceutique; 11 février 2011. Publié au http://www.vendredipm.ca/. Consulté le 7 novembre 2011.
17. Loi sur les contrats des organismes publics, L.R.Q., c. C-65.1. Publié au www2.publicationsduquebec.gouv.qc.ca/dynamicSearch/telecharge.php?t ype=2\&file=/C_65_1/C65_1.html. Consulté le 6 décembre 2010.

18. Hiltzik M. FDA needs teeth to avert drug shortages. LA Times; 19 avril 2011. Publié au http://articles.latimes.com/2011/apr/19/business/lafi-hiltzik-20110419. Consulté le 9 mai 2011.

19. Weeks C. The drug shortage hits home. Globe and Mail [Toronto, ON]; 30 janvier 2011. Publié au www.theglobeandmail.com/life/health/thedrug-shortage-hits-home/article1886802/. Consulté le 9 mai 2011.

20. Blackwell T. Drug-shortage survey a "wake-up call", pharmacists say. National Post [Toronto, ON]; 16 décembre 2010. Publié au http://news.nationalpost.com/2010/12/15/drug-shortage-survey-a-wakeup-call-pharmacists/. Consulté le 9 mai 2011.

21. Law $S$. Doctors, pharmacists seek online database to track drug shortage. Vancouver Sun; 6 mai 2011. Publié au www.vancouversun.com/ news/todays-paper/Doctors+pharmacists+seek+online+database+ track+drug+shortages/4737739/story.html. Consulté le 9 mai 2011.

22. Champagne S. Dix medicaments en rupture de stock. La Presse [Montréal, QC]; le 13 novembre 2010. Publié au www.cyberpresse.ca/vivre/ sante/201011/13/01-4342365-dix-medicaments-en-rupture-destock.php. Consulté le 9 mai 2011.

23. Nova Scotia drug shortage hurts health care. CBC News. Publié au www.cbc.ca/news/canada/nova-scotia/story/2011/01/06/ns-drug-shortage-antidepressant.html. Consulté le 9 mai 2011.

24. Baumer AM, Clark AM, Witmer DR, Geize SB, Vermeulen LC, Deffenbaugh JH. National survey of the impact of drug shortages in acute care hospitals. Am J Health Syst Pharm 2004;61(19):2015-2022.

25. Fox ER, Tyler LS. Managing drug shortages: seven years' experience at one health system. Am J Health Syst Pharm 2003;60(3):245-253.

26. Fox ER, Tyler LS. Measuring the impact of drug shortages [éditorial]. Am J Health Syst Pharm 2004;61(19):2009.

27. Fox E, Tyler LS. Recent trends in drug shortages: an update from the 2003 report. Am J Health Syst Pharm 2009;66(9):798-800.

28. Jensen V, Rappaport BA. The reality of drug shortages-the case of the injectable agent propofol. $N$ Engl J Med 2010;363(9):806-807.

29. Rapport annuel de gestion 2009-2010. Québec (QC) : Conseil du médicament; 2010. Publié au www.cdm.gouv.qc.ca/site/download.php? $\mathrm{f}=f 78 \mathrm{c} 2 \mathrm{f} 99 \mathrm{fc} 33711 \mathrm{fb} 711209 \mathrm{~d} 37 \mathrm{baf666}$. Consulté le 10 mai 2011.

30. Harding J. "Systèmes de distribution de médicaments ». Dans : Babich M, Bornstein C, Bussières JF, Hall K, Harding J, Lefebvre P, et coll., comité de rédaction. Rapport annuel 2009-2010 sur les pharmacies hospitalières canadiennes. Eli Lilly; 2010. Publié au http://www.lilly hospitalsurvey.ca/hpc2/content/rep_2010_tocF.asp. Consulté le 9 mai 2011.

31. Robert N. «Achat de médicaments et contrôle des stocks ». Dans : Babich M, Bussières JF, Hall KW, Harding J, Johnson N, Lefebvre P, et coll., comité de rédaction. Rapport annuel 2005-2006 sur les pharmacies hospitalières canadiennes. Éthique en pharmacie hospitalière. Eli Lilly; 2006. Publié au www.lillyhospitalsurvey.ca/hpc2/content/rep_2006_tocF.asp. Consulté le 9 mai 2011.

32. Apotex Inc. Warning letter. Dans : Inspections, compliance enforcement and criminal investigations. Silverspring (MD) : Food and Drug Administration; 25 juin 2009. Publié au www.fda.gov/ICECI/Enforcement Actions/WarningLetters/ucm170912.htm. Consulté le 10 mai 2011.

33. Apotex Inc. Warning letter. Dans : Inspections, compliance enforcement and criminal investigations. Silverspring (MD) : Food and Drug Administration; 29 mars 2010. Publié au www.fda.gov/ICECI/Enforcement Actions/WarningLetters/ucm207508.htm. Consulté le 10 mai 2011.

34. Apotex reprend ses livraisons vers les Etats-Unis. Montréal (QC) : CNW Telbec; 2011. Publié au www.cnw.ca/fr/releases/archive/May2011/ 10/c3134.html. Consulté le 11 mai 2011.

35. Thompson CA. Senator proposes drug shortages law. Am J Health Syst Pharm 2011;68(6):461.

36. Thompson CA. Stakeholders in supply chain discuss shortages. Am J Health Syst Pharm 2011;68(1):9-10. 
37. Provisional observations on drug product shortages: effects, causes, and potential solutions. Am J Health Syst Pharm 2002;59(22):2173-2182.

38. Weaver JM. Why are there so many drug shortages? Anesth Prog 2010;57(3):89-90.

39. Johnson TJ. Drug shortages: an increasing problem for patients and clinicians. S D Med 2011;64(1):14-15.

40. McKenna M. Hospital pharmacists scrambling amid vast drug shortages: emergency physicians between roc and a hard place. Ann Emerg Med 2011;57(2):A13-A15.

41. Jensen V, Kimzey LM, Goldberger MJ. FDA's role in responding to drug shortages. Am J Health Syst Pharm 2002;59(15):1423-1425.

42. Thompson CA. FDA improves communication about drug shortages. Am J Health Syst Pharm 2004;61(9):880,882.

Jean-François Bussières, B. Pharm., M.Sc., est chef du Département de pharmacie et de l'Unité de recherche en pratique pharmaceutique au CHU Sainte-Justine et professeur titulaire de clinique à la Faculté de pharmacie de l'Université de Montréal, Montréal, Québec.
Andrei Chiveri est assistant de recherche à l'unité de recherche en pratique pharmaceutique, CHU Sainte-Justine, Montréal, Québec.

Denis Lebel, B. Pharm., M.Sc., est adjoint aux soins pharmaceutiques, à l'enseignement et à la recherche et à l'unité de recherche en pratique pharmaceutique au CHU Sainte-Justine, Montréal, Québec.

\section{Adresse de correspondance :}

Jean-François Bussières

Département de pharmacie

Centre Hospitalier Universitaire Sainte-Justine

3175, chemin de la Côte Sainte-Catherine

Montréal QC H3T 1C5

Courriel : jf.bussieres@ssss.gouv.qc.ca

\section{Remerciements}

Les auteurs remercient Lionel Brisseau pour l'aide apportée à la collecte de données.

\section{CJHP Call for photographs}

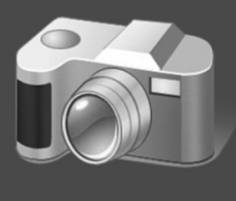

The Canadian Journal of Hospital Pharmacy is looking for photographs for the cover of the Journal.

The photo theme for 2012 is:

\section{Canadian Landscapes and Scenery}

Interested participants are asked to submit a digital photo or group of photos for selection along with a short ( $\max 150$ words) write-up about the location of the photo, the date and time of the photo, and the type of equipment used. Be sure to include any stories or details that make this photograph or location unique!

Entries can be submitted to Colleen Drake, Publications Administrator, at cdrake@cshp.ca.
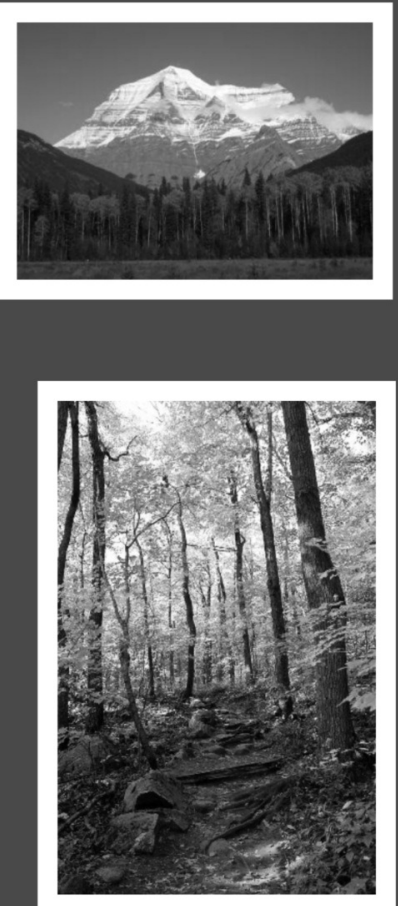